Trinity University

Digital Commons @ Trinity

Psychology Faculty Research

Psychology Department

$11-2003$

\title{
Transfer of Training Emotionally Biased Interpretations
}

Paula T. Hertel

TrinityUniversity, phertel@trinity.edu

A. Matthews

S. Peterson

K. Kintner

Follow this and additional works at: https://digitalcommons.trinity.edu/psych_faculty

Part of the Psychology Commons

Publication Details

Applied Cognitive Psychology

\section{Repository Citation}

Hertel, P.T., Mathews, A., Peterson, S., \& Kintner, K. (2003). Transfer of training emotionally biased interpretations. Applied Cognitive Psychology, 17(7), 775-784. doi: 10.1002/acp.905

This Article is brought to you for free and open access by the Psychology Department at Digital Commons @ Trinity. It has been accepted for inclusion in Psychology Faculty Research by an authorized administrator of Digital Commons@ Trinity. For more information, please contact jcostanz@trinity.edu. 


\title{
Transfer of Training Emotionally Biased Interpretations
}

\author{
PAULA T. HERTEL ${ }^{1} *$ ANDREW MATHEWS ${ }^{2}$, \\ SAMANTHE PETERSON ${ }^{1}$ and KATHERINE KINTNER ${ }^{1}$ \\ ${ }^{1}$ Trinity University, USA \\ ${ }^{2}$ Medical Research Council Cognition and Brain Sciences Unit, Cambridge, UK
}

\begin{abstract}
SUMMARY
Non-anxious college students first performed a semantic-judgement task that was designed to train either threat-related or threat-unrelated interpretations of threat-ambiguous homographs (e.g. mug). Next they performed an ostensibly separate transfer task of constructing personal mental images for single words, in a series that included new, threat-ambiguous homographs. In two experiments, the number of threat-related interpretations in the transfer task significantly increased following threatrelated experience during the training phase, compared to other training conditions. We conclude that interpretive biases typically shown by anxious people can be established in non-anxious students in ways that generalize to novel tasks and materials. Copyright (C) 2003 John Wiley \& Sons, Ltd.
\end{abstract}

One of the signs of anxiety disorder is a tendency to find threatening meaning in ambiguous situations. A noise in the middle of the night, the unsmiling glance from a colleague, and unanticipated laughter during one's lecture are examples of ambiguous events that anxious people tend to interpret in emotionally negative or threat-related ways. Interpretive biases have been demonstrated in the laboratory with materials ranging from homographs (words spelled the same but with different meanings) to social scenarios, and with participants experiencing high levels of trait anxiety as well as a variety of anxiety disorders (e.g. Eysenck et al., 1991; Hirsch \& Mathews, 2000; Richards \& French, 1992). Investigators have traditionally emphasized the role played by the ongoing anxious state in increasing sensitivity to threatening stimuli. Recently, however, the opposite direction of cause has received some attention. Threat-related biases have been established experimentally in the behavior of non-anxious people through repetitive experience that encourages threatrelated interpretations, and subsequent increases in state anxiety have been observed (see Mathews \& MacLeod, 2002, for review). This approach to understanding anxiety-related biases in interpretation by modelling it in the behaviour of non-anxious individuals calls attention to the role of experience in developing and maintaining anxiety disorders.

In a series of experiments described by Grey and Mathews (2000), systematic exposure to either emotional valence of a set of threat/non-threat homographs resulted in nonanxious participants tending to apply congruent interpretations to novel homographs presented within a subsequent task. For example, participants in Experiment 3 performed a 
series of relatedness judgements in which threat/non-threat homographs (e.g. fit) were each followed by a word that was always associated with either meaning valence (e.g. epileptic or healthy); unrelated pairs consisted of threat-neutral homographs and other words. Following such training, the participants performed a lexical-decision task in which old and new homographs served as primes. On some trials the target for lexical decision was a word associated with the threatening meaning of the priming homograph, and on other trials a threat-unrelated target appeared. The critical finding was that lexical decision times were influenced in the direction that was emotionally congruent with the bias established during the training phase, even following primes not seen in the training task. Thus, newly established interpretive habits generalized both to new ambiguous words and to a related but new task.

In a similar vein, Mathews and Mackintosh (2000) exposed non-anxious participants to self-related descriptions of social events with either threat-related or threat-unrelated outcomes and then to similar texts in which the outcomes remained ambiguous. In a later recognition test of items from the ambiguous texts, participants endorsed alternatives with meanings that matched the training bias (either threat-related or threat-unrelated bias characterizing the outcomes of the unambiguous scenarios). Furthermore, in experiments in which participants were required to generate the endings during the training phase (by completing word fragments for either threatening or nonthreatening conclusions), experience with threatening outcomes temporarily increased the level of anxiety, and experience with benign outcomes reduced it. These and other experiments (see Mathews \& MacLeod, 2002) demonstrate that induced emotional processing biases can have significant causal effects on reported anxiety, albeit only when the induction influences how emotionally significant information is interpreted.

In each of these reports, the bias established during training transferred to very similar tasks in the next phase. Building on these findings, we report two studies that show transfer of training biases beyond the tasks in which the biases were initially practiced. Nonanxious students were randomly assigned to develop habits of interpreting homographs in either threat-related or threat-unrelated ways. In that sense they were trained to interpret in one or the other way, although no awareness of the manipulation was intended to occur. Then they participated in a task described as a pilot study concerning the construction of mental images. Recordings of their descriptions of the images allowed us to evaluate their interpretations of new homographs included among the materials (none of which was presented during training). Compared to the tasks previously used, this transfer task is closer to the kinds of relatively unstructured situations that occur naturally in the course of encountering ambiguity. Because it also has very little in common procedurally with the training task, evidence of transfer provides greater generalization and thereby serves as an important next step in the experimental analysis of biased interpretations common among anxious people.

\section{EXPERIMENT 1}

\section{Method}

Overview of design

Non-anxious participants were randomly assigned to undergo a training phase that encouraged either threat-related or threat-unrelated interpretations of a set of threat-ambiguous 
homographs (e.g. stroke, plot, patient) or to bypass the training phase entirely (no-training control). In the transfer phase, all participants were instructed to construct mental images corresponding to a series of new words, some of which were threat-ambiguous homographs. We recorded their descriptions of each image and classified the use of the homographs as threat-related, threat-unrelated, or ambiguous.

\section{Participants}

A total of 80 undergraduate students at Trinity University volunteered to participate in exchange for extra credit in their introductory course. At the beginning of each session, the participant completed a mood form to indicate current feelings as well as feelings during the past week. Students who rated their current level of anxiety or their current level of distress above 4 on the 7-point scale (or who rated their level during the past week above 5) were assigned to a pseudo-anxious control condition $(n=26)$ and completed only the transfer phase (in order to obtain some experimental credit). Their data were not included as a separate condition in the analyses because our measure of anxiety was a 'rough' one. The remaining participants were randomly assigned to one of the three training conditions (threat, non-threat, and no-training control), subject to the constraint of equal cell sizes and gender distribution; 12 women and 6 men participated in each condition.

\section{Materials}

Mood and anxiety forms. At the top of the mood form used at the start of the session, participants were asked to rate the extent to which their feelings 'at this moment' corresponded to each of the adjectives listed below (alert, distressed, interested, distracted, content, anxious). A scale ranging from 1 (not at all) to 7 (extremely) was printed to the right of each adjective. At the bottom of the form, the same scales followed the instruction to rate the extent to which they were feeling each way during the past week. At the end of the session, we administered the 'state' version of the State-Trait Anxiety Inventory (STAI; Spielberger et al., 1983).

Training phase. On each trial two consecutively presented words were presented for a judgement of semantic relatedness. The second words, constant across the two training conditions, belonged either to a set of 40 critical homographs (with at least one threatrelated and one threat-unrelated meaning), to a set of 40 neutral homographs (without threat-related meanings), or to a set of 20 non-homograph fillers (also without threatrelated meanings). An example of a critical homograph is punch (which refers to a drink or a fighting movement). The critical homographs were adapted for US usage from those selected by Grey and Mathews (2000), according to the ratings obtained by French and Richards (1992) for threatening and nonthreatening meanings. The neutral homographs were also taken from Grey and Mathews (2000); examples include pen, and trace. The fillers (e.g. basement, fact, geese) were chosen from among those words rated 3 or lower for emotionality (on a 7-point scale) on the norms obtained by Rubin and Friendly (1986). These 100 words - always the second to occur in the trial-were repeated for an additional 100 trials. Each word was preceded by two different words on the two occasions of its occurrence and, for the critical homographs only, both 'priming' words were of the emotional valence appropriate to that training condition.

All the priming words were selected from Nelson et al.'s (1998) association norms. The two priming words for 30 of the 40 neutral homographs were paired with nonassociated 
homographs from the same set (i.e. prime-homograph associates were rearranged) to constitute trials on which the relatedness judgement should be 'no'. The same was done for 15 of the 20 filler words. The priming words for the 40 critical homographs were always presented prior to the associated homograph; threat-related primes were chosen for the threat-training condition and threat-unrelated primes for the non-threat-training condition (e.g. for punch: fist and fight versus drink and bowl). The threat ratings for these critical primes were balanced across the two sets of 100 trials, and the lists for the two training conditions were balanced for frequency and number of letters. The order of trials was randomized within blocks of five, with two critical homographs, two neutral homographs, and one filler in each block.

Transfer phase. A list of 16 threat/non-threat homographs (not used in the training phase), 16 non-homographs, and four fillers (two primacy and two recency buffers) was compiled from the norms by French and Richards (1992) and Rubin and Friendly (1986), the latter to ensure low emotionality for the non-homographs. The list is presented in the Appendix. Although we lack normative frequency data for the separate meanings of the homographs, we chose ones that produced numbers of associates in the study by French and Richards (1992) that were approximately equal on average for 14 of the 16 homographs $(M=60$ threat related associates and 62 threat-unrelated associates). The other two homographs were insufficiently close in meaning to count (strike instead of striking and battery instead of batter). Half of those 14 homographs produced threat-related responses that were more prevalent than were threat-unrelated responses in their study.

\section{Procedure}

The session was described as a collection of independent tasks (decisions about word meanings, construction of mental images for words, forms concerning personal characteristics), each too short to justify credit by itself. After informed consent, participants were asked to fill out the brief mood form. In taking the completed form, the experimenter mentioned that she must determine whether the participant was needed for all remaining tasks and would return in a moment (after actually having determined whether the participant was eligible for random assignment to a training condition).

Training phase. The procedure for training was borrowed from Grey and Mathews (2000, Experiment 3). The materials for all phases were presented in bold black font on a light grey screen, programmed in Superlab Pro for a Pentium processor. On each trial of the training phase, the priming word appeared just above center screen and remained on screen when the second word appeared just below center, $750 \mathrm{~ms}$ later. The two words remained on screen until the participant responded. The ' $\mathrm{v}$ ' key on the keyboard was labelled 'yes' and the 'n' key 'no'. A 1-min break separated sets of 100 trials. Participants were instructed to respond as quickly as possible, without sacrificing accuracy.

Transfer phase. This task was described as a pilot study for selecting materials to be used in future experiments on mental images. On each trial, the participants were asked to do three things: (a) form a mental image of the word that included the participant, (b) describe that image in a sentence to be spoken aloud and recorded, and (c) rate the degree and valence of emotion associated with the image, on a scale of 1 (very positive) to 9 (very 
negative). Each of the 32 words (preceded and followed by two buffers) was displayed for $10 \mathrm{~s}$, during which time the participants created the image and reported the sentence. During instruction, this process was illustrated with the example of beach. They might imagine themselves walking along a beach and then describe the image by saying, 'I am walking along a beach in the summer'. After $10 \mathrm{~s}$ the rating scale replaced the word on the screen. The trial ended when the rating was typed and the program directly advanced to the next item. Right before starting the program, the experimenter turned on the tape recorder and left the room. Participants were told that someone other than the experimenter would eventually listen to the tape to classify the sentences, but that their identity would not be disclosed and the sentences would not be reported anywhere. At the end of the session, prior to debriefing, participants were asked to fill out the 'state' part of the STAI and to seal it anonymously in an envelope to be opened by the supervising faculty member.

\section{Results and discussion}

For all analyses in both experiments, the significance level was set at 0.05 . Initial analyses were performed with gender as a factor; because this grouping variable did not significantly interact with the other factors, it was omitted in the reported analyses.

\section{Training errors}

Due to a mishap, training data were missing for seven participants in each condition. Judgement errors (defined as responses opposite to the predetermined 'correct' response) made by the remaining 22 participants occurred on an average of $7.5 \%$ of the trials and did not significantly vary according to the type of item or the training condition (results similar to those obtained by Grey \& Mathews, 2000).

\section{Interpretations}

The sentences produced in response to each of the 16 homographs presented in the transfer phase were evaluated independently by two judges, blind to training conditions, who listened to the tapes of eight participants. They classified the usage of the homographs as threat-related, threat-unrelated, or ambiguous, based on the context provided by the sentence (e.g. respectively for the homograph battery: I read about the assault and battery/ I bought a new battery for my car/ I saw the battery). Average agreement was 94\%. Disagreement almost always concerned the classification of one particular homograph. With that ambiguity clarified, the remainder of the tapes was scored by one of the judges (who was not part of the research team that designed the study and collected data). The tape from one female participant in the non-threat-training condition was frequently inaudible, so her data are missing from the analysis.

The numbers of sentences produced by the participants were submitted to an analysis of variance, with a between-subjects factor for training condition (threat, non-threat, or none) and a within-subjects factor for sentence meaning (threat-related and threat-unrelated, as classified from the tapes). Means and standard deviations are reported in the top half of Table 1. Figure 1 illustrates the significant interaction, $F(2,50)=4.56, M S E=5.66$, $p<0.02$. When the numbers of threat-related and threat-unrelated interpretations were examined separately, each analysis revealed a significant overall effect of training (across the three conditions) and a significant difference between the two actual training conditions. The mean number of threat-related interpretations was higher in the threat 
Table 1. Mean number of homograph interpretations in the transfer phase (standard deviation)

\begin{tabular}{lccr}
\hline Training condition & $n$ & Threat related & Threat unrelated \\
\hline Experiment 1 & & & \\
$\quad$ Threat & 18 & $6.7(1.28)$ & $8.6(1.38)$ \\
$\quad$ Non-threat & 17 & $5.0(1.50)$ & $10.2(1.68)$ \\
$\quad$ Control & 18 & $5.6(2.00)$ & $10.0(2.22)$ \\
Experiment 2 & & & \\
$\quad$ Threat & 22 & $5.7(1.28)$ & $9.3(1.58)$ \\
$\quad$ Non-threat & 22 & $4.1(1.60)$ & $11.0(1.90)$ \\
\hline
\end{tabular}

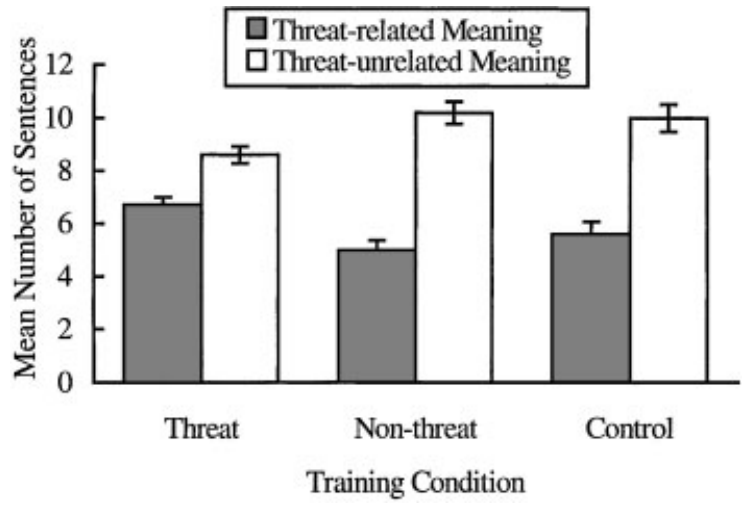

Figure 1. The mean number of interpretations that were judged to be related or unrelated to threat, during the transfer phase of experiment 1 . Error bars represent one SE

trained condition than in the non-threat trained condition, $t(50)=3.03, S E=0.55$, $p<0.01$. The mean number of threat-unrelated interpretations was higher in the nonthreat trained condition than in the threat trained condition, $t(50)=-2.67, S E=0.61$, $p<0.01$. In addition, the threat trained condition, compared to the no-training controls, produced fewer threat-unrelated interpretations, $t(50)=-2.32, S E=0.60, p<0.025$, and a trend toward more threat-related interpretations, $t(50)=1.95, S E=0.54, p<0.06$.

Results from the main analysis also confirmed the obvious - a main effect of type of interpretation. Across groups, $60 \%$ of sentences reflected the threat-unrelated meaning of the homographs. So, it is clear that the effect of training threat-related interpretations was mainly one of reducing that tendency to interpret in non-threatening ways.

\section{Anxiety scores}

The mean state-anxiety score on the STAI was 37 in the threat condition, 39 in the nonthreat condition, and 38 for no-training controls. No significant differences obtained, $F<1.0$.

\section{EXPERIMENT 2}

Experiment 2 was conducted primarily as an attempt to replicate (and to produce a full set of training data), but we made several additional changes. First, after considering the possibility that the ratings of emotionality in the interpretation phase might have called 
attention to the nature of the homographs by establishing demand to construct at least some emotional images, we changed the rating dimension to vividness of the image constructed for each item. Second, because some participants in Experiment 1 occasionally took many seconds to make relatedness judgements during the training phase, we attempted to reduce the number of such trials by inserting a request for speeding up judgements at the end of trials longer than $3 \mathrm{~s}$. Third, we restricted participation to those who scored below 40 during a prescreening administration of the trait version of the STAI. Fourth, due both to the difficulties of recruiting and to the clarity of the control-group data in Experiment 1, we eliminated the no-training control condition. Last, the materials were revised.

\section{Method}

\section{Participants and design}

Participants for Experiment 2 were prescreened on the STAI, administered to introductory psychology classes. Those who scored below 40 on the Trait form were phoned and invited to participate, although no linkage with the STAI was mentioned. Those who agreed to participate $(N=46)$ were randomly assigned to receive threat or non-threat training, subject to the constraint of equal cell sizes balanced for gender. The data from two participants were replaced, due to experimenter error, and the data from 14 women and 8 men in each training condition constituted the final sample.

\section{Materials and procedure}

Using the same guidelines and norms as used in Experiment 1, we revised the materials for both training and transfer phases. (The Appendix lists the words used in the transfer phase.) Procedures were also identical to those in Experiment 1, with three exceptions. First, made unnecessary by our prescreening on the STAI, the brief mood forms were no longer used. Second, if the participant took longer than $3 \mathrm{~s}$ to make a relatedness judgment during the training phase, 'try to respond faster' appeared in large red font at the end of the trial. Third, we replaced the rating for emotionality, used as a cover story during the transfer phase in Experiment 1, with a rating for the vividness of the image. Participants were instructed to use 1 for images that were not at all clear and very poorly formed, 7 for images that were extremely vivid in their memory or imagination, and the numbers from 2 to 6 for images between those extremes.

\section{Results and discussion}

\section{Training errors}

The percentages of errors during training were submitted to an analysis of variance, with a between-subject factor for training condition and a within-subject factor for the type of trial (critical, threat-ambiguous homographs versus other). More errors were made on the critical trials $(M=15.6 \%)$ than on other trials $(M=7.5 \%), \quad F(1,42)=56.17$, $M S E=26.16, p<0.001$. Although this effect did not depend on the training condition, $F<1.0$, a marginally significant main effect of training condition was found, $F(1,42)=3.56, M S E=21.53, p<0.07$. Furthermore, the analysis of the percentage of errors across all trials, not sorted according to the type of trial, revealed a significant effect of training condition, $t(42)=2.02, S E=0.92, p<0.05,(M=11.7 \%$ in the threat condition versus $9.8 \%$ in the non-threat condition). The increase in error rate, in comparison to Experiment 1 probably reflects the change in procedure by introducing warnings to speed up. 


\section{Interpretations}

As in Experiment 1, the numbers of sentences produced by the participants were submitted to an analysis of variance, with a between-subjects factor for training condition (threat versus non-threat) and a within-subjects factor for sentence meaning (threat-related and threat-unrelated). Means and standard deviations are reported in the bottom half of Table 1 . A significant interaction again emerged, $F(1,42)=13.03, M S E=4.78, p<0.001$. When the numbers of threat-related and threat-unrelated interpretations were examined separately, each analysis revealed a significant difference between the two training conditions. The threat trained participants (compared to non-threat trained) produced a higher mean number of threat-related interpretations, $t(42)=3.75, S E=0.44, p<0.001$, and fewer threat-unrelated interpretations, $t(42)=-3.28, S E=0.53, p<0.002 .^{1}$

As in Experiment 1, a significant main effect of type of interpretation was obtained. Across groups, $63 \%$ of sentences reflected the threat-unrelated meaning of the homographs. The pattern was similar to the pattern in Experiment 1, and so the effect of training threat interpretations of this modified set of homographs seemed to be one of reducing the tendency to interpret in non-threatening ways. The absence of a no-training control group, however, makes this conclusion tentative.

\section{Anxiety scores}

The mean STAI state score for participants trained for threat was 43 , versus 40 for those trained to interpret threat-unrelated meanings. The difference was non-significant, $t<1.0$.

\section{GENERAL DISCUSSION}

In both experiments, the experimentally established habit of interpreting ambiguity in either threat-consistent or inconsistent ways carried over into a task of constructing mental images corresponding to other ambiguous words. In Experiment 1, participants trained to resolve ambiguity in the direction of threat produced more threat-related interpretations in the transfer task than did those who were trained to perceive threat-unrelated meaning and those who did not undergo a training phase. The training difference was replicated in Experiment 2, although the absence of a no-training control group makes it difficult to determine the precise nature of the bias in that experiment.

The bias established during training in these experiments played a role during the transfer phase that is similar to the role of prior knowledge in text comprehension. We have long known that the interpretation of ambiguous text is easily influenced by the provision of direct links to prior knowledge (e.g. Bransford \& Johnson, 1972) or advice to adopt a well-understood perspective (e.g. Pichert \& Anderson, 1977), but the links in our study were not direct. Yet, even indirect links — such as superficial similarity to one or another recently read passage with differential relevance to the text at hand-have been shown to affect interpretation (Ross \& Bradshaw, 1994). In the experiment by Ross and Bradshaw (1994), remindings through superficial similarity were accomplished without

\footnotetext{
${ }^{1}$ The percentage of errors during training was used as a covariate in an analysis of covariance performed on the number of threat-related interpretations. The covariate accounted for a significant component of variance, $F(1,41)=4.63, M S E=1.93, p<0.05$. A greater number of threat-related interpretations was produced by those who made more errors, $r(42)=0.41$. The effect of training condition was also significant, in the direction reported for the previous analysis, $F(1,41)=9.54, p<0.004$.
} 
the participants' intent to use the prior information. Similarly, none of our participants reported awareness of the link between the training and transfer phases. Then, in what sense were our participants 'reminded' of their recently trained tendency to perceive threat-related meaning more often than they might otherwise do? Sufficient basis for transfer perhaps was located in the common act of evaluating the meaning of words presented in an experimental setting. In much the same way, everyday situations involving some degree of ambiguity (e.g. social events) may provide cues that elicit interpretations previously practised. If threat-related interpretations are practised, they can transfer to similar everyday situations and perhaps contribute to anxious mood states. Our study provides merely a first step in generalization to novel situations. Full application awaits evidence of transfer to events even more removed in time and space from the training experience.

Moreover, given the absence of effects on state anxiety, a full application to interpretative biases in anxious states must rest on continued research in this domain. In previous research on training biases akin to those found in anxiety, evidence of increased levels of state anxiety has been found, but only under certain conditions (see Mathews \& MacLeod, 2002). For example, in the experiments reported by Mathews and Mackintosh (2000) shifts in state anxiety scores failed to occur unless the participants were engaged in producing the threat-related endings of the passages. In considering those results, the lack of an effect on anxiety scores in our study was not surprising; the average number of threat-related images produced by our participants was about six - many fewer trials than the 80-100 generated-ending trials in the experiments by Mathews and Mackintosh (2000). Furthermore, our 80 training trials did not invite active generation. Thus, an effect on reported anxiety has not been found when participants have minimal experience in resolving ambiguity in threat-related ways.

With more experience, increases in anxiety might begin to emerge. We speculate that, across unrelated situations in the real world, novel ambiguous cues invite interpretations congruent with previous understandings and consequently cause at least momentary increases in anxiety. For example, prior thinking about the possible disapproval of others may be sufficient to influence the interpretation of a colleague's half-smile as more derogatory than friendly. Prior thinking about the possibility of contracting a disease may establish the perception of a later bodily sensation as more ominous than transitory. And because prior thinking about such matters - replete with generative experienceprobably has induced anxiety, it is also likely that the 'transfer task' gives rise to momentarily anxious affect. The part of this picture that has been addressed by the present results is this: We now know that interpretations of the same sort as those made by anxious individuals (and demonstrated in a variety of experiments for the past several decades) can be trained to occur as non-anxious students face a new task with new materials. This evidence, together with other recent studies in which anxiety has been experimentally elevated, constitutes support for the depiction of anxiety as reaction to biased habits of interpretation.

\section{ACKNOWLEDGEMENTS}

We acknowledge the assistance of Faith Brosovich, Nissa Hillin, Austin Houck, Talina Miele, Jessica Riedel and Julie Vaughan, who helped prepare materials, collected data, or served as judges of interpretations. 


\section{REFERENCES}

Bransford, J. D., \& Johnson, M. K. (1972). Contextual prerequisites for understanding: some investigations of comprehension and recall. Journal of Verbal Learning and Verbal Behavior, 11, 717-726.

Eysenck, M. W., Mogg, K., May, J., Richards, A., \& Mathews, A. (1991). Bias in interpretation of ambiguous sentences related to threat in anxiety. Journal of Abnormal Psychology, 100, 144-150.

French, C. C., \& Richards, A. (1992). Word association norms for a set of threat/neutral homographs. Cognition and Emotion, 6, 65-87.

Grey, S., \& Mathews, A. (2000). Effects of training on interpretation of emotional ambiguity. Quarterly Journal of Experimental Psychology, 53, 1143-1162.

Hirsch, C., \& Mathews, A. (2000). Impaired positive inferential bias in social phobia. Journal of Abnormal Psychology, 109, 705-712.

Mathews, A., \& Mackintosh, B. (2000). Induced emotional interpretation bias and anxiety. Journal of Abnormal Psychology, 109, 602-615.

Mathews, A., \& MacLeod, C. (2002). Induced processing biases have causal effects on anxiety. Cognition and Emotion, 16, 331-354.

Nelson, D. L., McEvoy, C. L., \& Schreiber, T. A. (1998). The University of South Florida word association, rhyme, and word fragment norms. http://www.usf.edu/FreeAssociation/

Pichert, J. W., \& Anderson, R. C. (1977). Taking different perspectives on a story. Journal of Educational Psychology, 69, 309-315.

Richards, A., \& French, C. C. (1992). An anxiety-related bias in semantic activation when processing threat/neutral homographs. Quarterly Journal of Experimental Psychology, 45, 503-525.

Ross, B. H., \& Bradshaw, G. L. (1994). Encoding effects of remindings. Memory \& Cognition, 22, 591-605.

Rubin, D. C, \& Friendly, M. (1986). Predicting which words get recalled: measures of free recall, availability, goodness, emotionality, and pronouncability for 925 nouns. Memory \& Cognition, 14, 79-94.

Spielberger, C. D., Gorsuch, R. L., Lushene, R., Vagg, P. R., \& Jacobs, G. A. (1983). Manual for the State-Trait Anxiety Inventory. Palo Alto, CA: Consulting Psychologists Press.

\section{APPENDIX: WORDS IN THE TRANSFER TASK}

\begin{tabular}{|c|c|c|c|}
\hline \multicolumn{2}{|c|}{ Experiment 1} & \multicolumn{2}{|c|}{ Experiment 2} \\
\hline Homographs & Non-homographs & Homographs & Non-homographs \\
\hline Blow & Barrel & Blow & Barrel \\
\hline Stalk & Doughnut & Stalk & Broccoli \\
\hline Battery & Shut & Plot & Send \\
\hline Lash & Spinach & Lash & Height \\
\hline Hang & Vacuum & Break & Lift \\
\hline Faint & Tool & Stroke & Tool \\
\hline Growth & Amount & Growth & Amount \\
\hline Stamp & Chew & Arms & Chew \\
\hline Mug & Cotton & Mug & Drive \\
\hline Leaves & Win & Leaves & Jump \\
\hline Beat & Moss & Beat & Read \\
\hline Choke & Fabric & Sentence & Fabric \\
\hline Part & Iron & Part & Ability \\
\hline Bark & Pole & $\mathrm{Gag}$ & Pole \\
\hline Sink & Foam & Sink & Foam \\
\hline Strike & Grass & Patient & Wit \\
\hline
\end{tabular}

